\title{
RE J1255+266-Detection of an Extremely Bright EUV Transient
}

\author{
MICHAEL DAHLEM
}

Space Telescope Science Institutet, 3700 San Martin Drive, Baltimore, MD 21218, USA

During a pointed ROSAT observation in the direction of the Coma cluster of galaxies an exceptionally bright EUV source, RE J1255+266, was detected serendipitously. The source is located close to the Galactic North pole, at $b_{I I} \simeq 89^{\circ}$. Its observed EUV flux $(62-110 \mathrm{eV})$ at the time of the detection was of order $7 \times 10^{-9} \mathrm{ergs} \mathrm{s}^{-1} \mathrm{~cm}^{-2}$, making RE J1255+266 temporarily one of the brightest EUV sources on the sky.

The EUV flare of RE J1255+266 has a light curve with a decay time of about 0.86 days. With respect to earlier non-detections, the source brightened by a factor of $>7000$. Such a behavior has not been observed before. Thus, it is unclear what type of source RE J1255+266 might be. The most likely optical counterpart is a faint $(V \sim 18.5 \mathrm{mag})$ object with a blue spectrum (taken from an objective-prism Schmidt plate). For more details on the optical identification see the paper by J. Pye (this conference).

Simultaneous observations with CGRO/BATSE resulted in non-detections of the source in the $8-50 \mathrm{keV}$ energy range.

\section{Introduction}

I report here on the serendipitous detection of a transient EUV source with the ROSAT Wide-Field Camera (WFC). The WFC is an EUV camera with its own optics onboard ROSAT. It is an independent instrument, aligned to the optical axis of the X-ray telescope (XRT). The WFC field of view (FOV) is $5^{\circ}$ across, compared to $2^{\circ}$ (PSPC) and $40^{\prime}$ (HRI). It works in the energy range of 17-210 eV, depending on the selection of one out of four filters. For more details see Barstow \& Willingale (1988) and Briel et al. (1994).

\section{Detection with the ROSAT WFC}

RE J1255+266 was detected during a ROSAT pointed observation near the eastern boundary of the Coma cluster of galaxies on June 25-July 7, 1994. The source was seen during several satellite orbits. The total integration time of the pointing is 16.74 ks. At an off-axis angle of $2^{\circ} .2$ the WFC registered a very bright source at $\alpha, \delta(2000)=$ $12^{h} 55^{m} 07^{s} .6,+26^{\circ} 41^{\prime} 21^{\prime \prime} \pm 1^{\prime}$ which would have been missed with the primary detector used for the pointing, the ROSAT High-Resolution Imager. Since the source could not be identified with any known object we named it-according to the IAU convention-RE J1255+266 (Dahlem \& Kreysing 1994).

\section{Other Observations}

Simultaneous observations with the Compton Gamma-Ray Observatory BATSE instruments (Fishman et al. 1989) resulted in non-detections by both the Large Area Detectors and the spectrometers, which sets upper limits to the 8-50 keV flux of RE J1255+266 during the EUV outburst (Dahlem et al. 1995; D95).

Follow-up radio observations with the VLA at 1.4 and $4.9 \mathrm{GHz}$ on September 29, 1994, also resulted in non-detections. The same holds for earlier observations toward RE

† Affiliated with the Astrophysics Division in the Space Science Department of ESA 


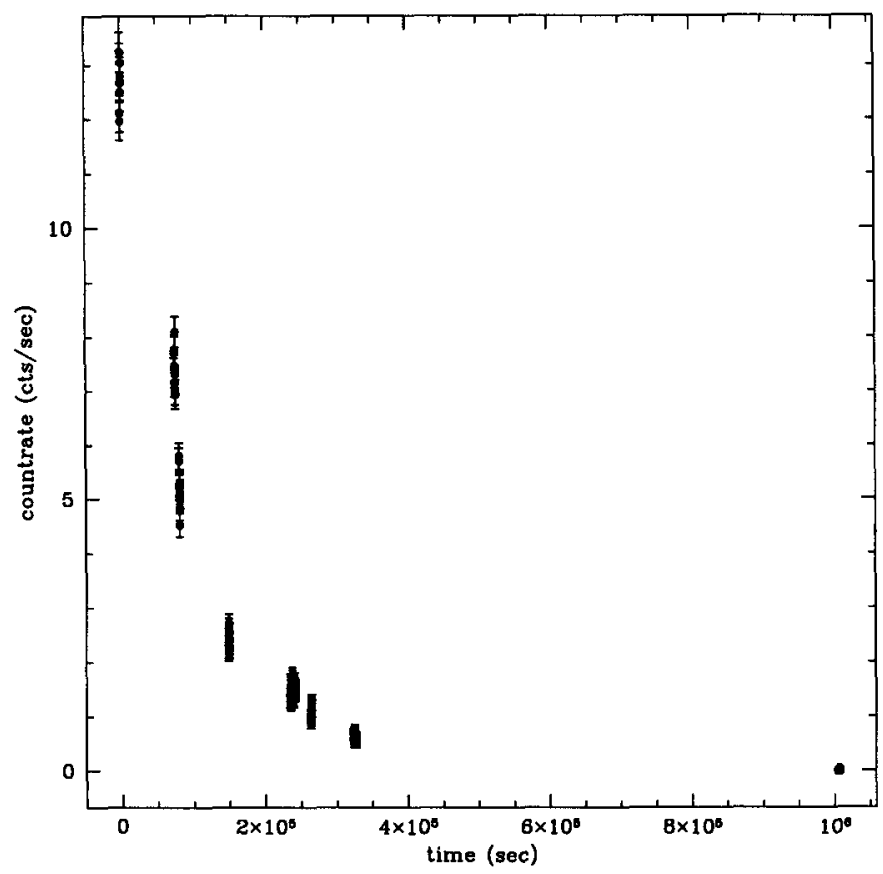

FIGURE 1. EUV light curve of RE J1255+266, binned into 100 second intervals. The observations started on June 25, 1994, 12:17 UT, and ended on July 7, 1994, 04:31 UT.

J1255+266 (D95), including the all-sky surveys of both ROSAT (WFC and PSPC) and EUVE (Pounds et al. 1993; Voges 1992; Bowyer et al. 1994).

A deep objective-prism Schmidt plate from the Hamburg Quasar Survey (HQS; Engels et al. 1988) shows a source with a blue spectrum about $1^{\prime} .3 \mathrm{NE}$ of the WFC position, spatially coincident with a very faint pointlike object on the POSS plate at $\alpha, \delta(2000)=$ $12^{h} 55^{m} 10^{s} .7,+26^{\circ} 42^{\prime} 28^{\prime \prime} \pm 1^{\prime \prime}$. D95 found this object to be the most likely optical counterpart of RE J1255+266. Follow-up observations by Watson (1995) corroborate this initial assumption. His results are summarized by Pye (this conference).

\section{The Unusual Properties of RE J1255+266}

Besides the fact that it is the first EUV transient-and the only one observed so far with good signal-to-noise-RE J1255+266 exhibits several characteristics which have not been observed before in transients that were detected in other wavebands.

RE J1255+266 was detected when its count rate in the $62-110 \mathrm{eV}$ band (WFC filter S2; Pounds et al. 1993) was about 14 counts $\mathrm{s}^{-1}$. At this time it was one of the brightest EUV sources on the sky. The sensitivity of the WFC at that time with respect to the all-sky survey in 1990 was 0.187 . Thus, at the time of the sky survey, the maximum count rate would have been 76.5 counts $\mathrm{s}^{-1}$, i.e., twice as bright as $\mathrm{HZ} 43$, the brightest source at this energy during the WFC survey (Durisen et al. 1976; Pounds et al. 1993). Lacking spectral information we must adopt a spectral slope, assuming a thermal plasma of, e.g., $T=2 \times 10^{5} \mathrm{~K}$ and an absorbing column density of $N(H)=10^{19} \mathrm{~cm}^{-2}$ for the 
conversion of count rates to flux units. At the time of the all-sky survey a count rate of $1 \mathrm{~s}^{-1}$ corresponded to $5-7 \times 10^{-11} \mathrm{ergs} \mathrm{s}^{-1} \mathrm{~cm}^{-2}$. Scaling this by the sensitivity loss since then $(1 / 0.187)$, the flux at the time of the detection was roughly

$$
f_{E U V}=0.3-1.1 \times 10^{-8}\left[\mathrm{ergs} \mathrm{s}^{-1} \mathrm{~cm}^{-2}\right] .
$$

Taking the mean value, this very high flux leads to a luminosity of

$$
L_{E U V} \sim 8.6 \times 10^{29} \frac{D^{2}}{\left[\mathrm{pc}^{2}\right]}\left[\operatorname{ergs~s}^{-1}\right] .
$$

For $N(H)=10^{18} \mathrm{~cm}^{-2}$ the flux would be a factor of 2 to 3 lower. The $N(H)$ values adopted above are only fractions of the total column density measured in HI line emission, $N(H)_{G a l}=8.676 \times 10^{19} \mathrm{~cm}^{-2}$ (Hartmann \& Burton 1995).

Figure 1 shows the unusual light curve of RE J1255+266. The half-light time of the (exponential) decay is $t_{1 / 2}=0.86 \pm 0.04$ days (D95). This is much shorter than the decay times of novae or dwarf novae (e.g., Kwok \& Leahy 1984, Richter 1992). In comparison with long-duration stellar flares of up to a few hrs (cf. Schmitt 1994) the decay time of the outburst observed in RE J1255+266 is much longer. Decay times of order one day are only observed in RS CVn systems.

Also the brightening factor of $>7000$ (D95) is unprecedented. This value we obtained by comparing our WFC pointing with non-detections during an earlier deep ROSAT pointing and the WFC and $E U V E$ all-sky surveys.

These characteristics of RE J1255+266 do not resemble the properties of any known class of objects. Thus, further observations or theoretical calculations are needed in order to clarify the nature of this bizarre source.

It is a pleasure to thank H.-C. Kreysing, S. M. White, C. Kouveliotou, and D. Engels for their contributions to this paper.

\section{REFERENCES}

Barstow, M. A. \& Willingale, R. 1988, Journal of the Brit. Interplan. Soc., 41, 345

Bowyer, S., Lieu, R., Lampton, M., Lewis, J., Wu, X., Drake, J. J., \& Malina, R. F. 1994, ApJS, 93, 569

BrIEL, U. G. ET AL. 1994, The ROSAT Users' Handbook, MPE Garching

Dahlem, M. \& Kreysing, H. -C. 1995, IAU Circ., 6085

DAHLEM, M. ET AL. 1995, A\&A, 295, L13; D95

Durisen, H. R., Savedoff, M. P., \& VAN, Horn, H. M. 1976, ApJ, 206, L149

Engels, D. Groote, D., Hagen, H. -J., \& Reimers, D. 1988, Optical Surveys for Quasars, ed. P. S. Osmer et al., Astr. Soc. of the Pacific Conf. Ser. 2, 143

Fishman, G. J. Et AL. 1989, Proc. Gamma Ray Observatory Science Workshop, NASA, Washington, 2

Hartmann, D. \& Burton, W. B. 1995, The Leiden-Dwingeloo Atlas of Galactic Neutral Hydrogen, Cambridge Univ. Press in preparation

KwoK, s. \& LEAHY, D. A. 1984, ApJ, 283, 675

Pounds, K. A. ET AL. 1993, MNRAS, 260, 77

PYE, J. 1995, This proceedings.

RichTer, G. A. 1992, Reviews in Modern Astronomy, 5, 26

SchmiTT, J. H. M. M. 1994, ApJS, 90, 735 
Voges, W. 1992, Space Science with Particular Emphasis on High Energy Astrophysics, Proc. of the European International Space Year Meeting, Munich, 9

Watson, M. G. 1995, Proc. of the Cape Workshop on Magnetic Cataclysmic Variables, Cape Town, January 23 submitted 